\title{
Unregulated long non-coding RNA-AK058003 promotes the proliferation, invasion and metastasis of breast cancer by regulating the expression levels of the $\gamma$-synuclein gene
}

\author{
$\mathrm{KAI} \mathrm{HE}^{1}$ and PEILIN WANG ${ }^{2}$ \\ ${ }^{1}$ Department of Surgery, The University Hospital of Shandong University, Jinan, Shandong 250100; \\ ${ }^{2}$ Department of General Surgery, The Second Hospital of Shandong University, Jinan, Shandong 250033, P.R. China
}

Received June 13,2014; Accepted December 18, 2014

DOI: $10.3892 /$ etm.2015.2323

\begin{abstract}
The aim of the present study was to investigate the function of long chain non-coding RNA (lncRNA) in breast cancer cells. Quantitative polymerase chain reaction was used to measure mRNA expression levels in breast cancer tissues, adjacent tissues and in MCF-7 breast cancer cells. Western blot analysis was used to determine the protein expression levels. In addition, a 3-(4,5-dimethylthiazol-2-yl)-2,5-diphenyltetrazolium bromide assay was employed to measure the rates of cell proliferation. The invasion and migration of the MCF-7 cells were examined using a Transwell ${ }^{\circledR}$ assay. The expression levels of IncRNA-AK058003 were increased significantly in the breast cancer tissues and were found to strongly correlate with the severity of the breast cancer clinical stage. Bioinformatics analysis revealed that the $\gamma$-synuclein gene (SNCG) may be a target gene regulated by lncRNA-AK058003. Thus, lncRNA-AK058803 was downregulated using small interfering RNA, and the mRNA and protein expression levels of SNCG were shown to be significantly reduced. Furthermore, the proliferation, invasion and migration rates of the MCF-7 breast cancer cells were significantly reduced. Therefore, the results demonstrated that unregulated lncRNA-AK058003 in breast cancer cells promotes cancer cell proliferation, invasion and metastasis via the regulation of SNCG expression.
\end{abstract}

\section{Introduction}

Breast cancer is a common clinical condition with increasing morbidity and high mortality rates (1). Previous studies have suggested that the development of breast cancer is associated with inactivated tumor suppressor genes, dysfunctional

Correspondence to: Dr Kai He, Department of Surgery, The University Hospital of Shandong University, 91 Shanda North Road, Jinan, Shandong 250100, P.R. China

Email: hkhk333@163.com

Key words: breast cancer, long non-coding RNA-AK058003, $\gamma$-synuclein gene, small-interfering RNA signaling transduction pathways and other malfunctioning molecular signaling processes (2-4). With the development of chemotherapy, hormonal therapy, immunotherapy, gene therapy and other treatment technologies, the long-term survival of breast cancer patients has become possible. Breast cancer patients continue to succumb to the disease due to tumor metastasis, drug resistance and other reasons including hemorrhage, infection and recurrence (5). Therefore, finding novel biomarkers for use in the early diagnosis and treatment of breast cancer has become increasingly studied.

A high-throughput sequencing study revealed the existence of types of non-coding RNA in gene transcripts, including small non-coding RNA and long non-coding (lnc)RNA (6). LncRNA has a length of $>200$ nucleotides and a similar structure to mRNA, including a 5' cap and poly-A tail. However, lncRNA is unable to encode proteins due to the absence of an open reading frame (7). Increasing numbers of studies have indicated that lncRNA may play an important role in various cellular processes, including cell differentiation, proliferation and apoptosis $(8,9)$. Notably, a previous study revealed a novel function of lncRNA in the development of prostate cancer (10). In addition, long non-coding homeobox antisense intergenic RNA is reported to be a highly valuable clinical biomarker for the prognosis and early diagnosis of ovarian cancer (11).

The primary functions of lncRNA include genomic imprinting, chromatin remodeling, cell cycle regulation and transcriptional regulation of adjacent mRNA (13). The $\gamma$-synuclein gene (SNCG), also known as breast cancer-specific protein 1, encodes a synaptic protein that was first identified in breast cancer. The expression of SNCG has been closely associated with tumor invasion and metastasis in a number of cancer types, including breast, stomach and liver cancer, as well as the clinical staging of cancer and the extent of lymph node metastasis (14-16). Activation of SNCG expression, induced by demethylation, has been observed to stimulate cell invasion and metastasis by activating the mitogen-activated protein kinase signaling pathway and the phosphorylation of the activator protein-1 transcription factor (17). In addition, SNCG is able to induce resistance to chemotherapy by inhibiting the c-Jun N-terminal kinase signaling pathway.

The present study examined the expression levels of IncRNA-AK058003 in breast cancer tissues and the effects 
on cell proliferation, invasion and migration. In addition, the potential of IncRNA-AK058003 as a transcriptional regulator of SNCG was investigated, as well as the underlying mechanism of action.

\section{Materials and methods}

Tissue samples. Breast cancer and adjacent tissue samples were collected from 30 patients (age range, 36-68 years; mean age, 42.5 years; median age, 45) who had received a clinical and pathological diagnosis of breast cancer between December 2012 and December 2013. The distance between the tumor tissue edge and the adjacent tissue was $>5 \mathrm{~cm}$. Tissue samples were rinsed in saline and immediately frozen with liquid nitrogen for storage at $-80^{\circ} \mathrm{C}$. The pathological stage of the breast cancer tissues and the extent of lymph node metastasis were diagnosed by two pathologists. The tissue samples were divided into well-, moderately- and poorly-differentiated groups according to the degree of differentiation, and were further classified into lymph node metastasis (N1) or lymph node non-metastasis (N0) groups depending on the extent of metastasis. The study was approved by the Ethics Committee of Shandong University (Jinan, China) and informed consent was obtained from all the participants or their families.

Reagents. Total RNA extraction reagent TRIzol ${ }^{\circledR}$, Dulbecco's modified Eagle's medium (DMEM) and Lipofectamine ${ }^{\circledR} 2000$ were purchased from Invitrogen Life Technologies (Carlsbad, CA, USA). MCF-7 breast cancer cells were purchased from the Cell Bank of the Chinese Academy of Sciences (Shanghai, China). Fetal bovine serum was purchased from Gibco Life Technologies (Grand Island, NY, USA) and Transwell ${ }^{\circledR}$ chambers were purchased from Corning, Inc. (Tewksbury, MA, USA). A rabbit anti-human SNCG polyclonal antibody was purchased from Abcam (Boston, MA, USA) and a Reverse Transcription System kit was purchased from Chengdu Bo Ruike Biotechnology Ltd. (Chengdu, China). The SYBR ${ }^{\circledR}$ Green quantitative polymerase chain reaction (qPCR) reagent was obtained from Kapa Biosystems, Inc. (Wilmington, MA, USA).

Cell cultures. MCF-7 cells were cultured in DMEM, supplemented with $10 \%$ fetal bovine serum, $100 \mathrm{U} / \mathrm{ml}$ penicillin and $100 \mu \mathrm{g} / \mathrm{ml}$ streptomycin, in an incubator at $37^{\circ} \mathrm{C}$ with $5 \%$ $\mathrm{CO}_{2}$. One day prior to transfection, log-phase MCF-7 cells were seeded into 24 -well plates $\left(2 \times 10^{5} /\right.$ well $)$ and classified into control, small interfering (si)RNA-AK058003 interference, scrambled siRNA negative control (NC) and mock groups. Transfection was performed on the following day when the cells had reached $70 \%$ confluence. Lipofectamine ${ }^{\circledR}$ $2000(1 \mu \mathrm{l})$ and $1.5 \mu \mathrm{l}$ siRNA solution $(20 \mathrm{pmol} / \mu \mathrm{l}$; RiboBio Co., Ltd, Guangzhou, China) were added separately to two Eppendorf tubes containing $50 \mu 1$ Opti-MEM ${ }^{\circledR}$ I (Thermo Fisher Scientific, Waltham, MA, USA). After standing for $5 \mathrm{~min}$, the two solutions were combined and left to stand for a further $20 \mathrm{~min}$ at room temperature. The mixture was subsequently transferred to culture plates to incubate for $6 \mathrm{~h}$. The medium was then replaced with fresh, high-glucose DMEM, supplemented with $10 \%$ fetal bovine serum, prior to continued incubation. After 48 and $72 \mathrm{~h}$ of incubation, cells were collected for the determination of gene and protein expression levels.

Bioinformatics. SNCG is strongly associated with breast cancer and thus coding genes upstream and downstream of SNCG was searched to check for the existence of lncRNA Information regarding the genomic location of AK058003 (8.4 kb upstream of SNCG on the same chromosome 10) was obtained by searching the UCSC database (http://genome.ucsc.edu/). The details of coding genes that were within $50 \mathrm{~kb}$ upstream and downstream of AK058003 were obtained from GenBank (http://www.ncbi.nlm.nih.gov/genbank/). No other genes were analyzed in the present study.

$q P C R$. Total RNA of the breast cancer tissues was extracted using TRIzol ${ }^{\circledR}$, according to the manufacturer's instructions. A $1-\mu \mathrm{g}$ sample of the total RNA was used for the reverse transcription reaction and $1-\mu 1$ was used for qPCR. The relative expression levels were analyzed using the $2^{-\Delta \Delta \mathrm{Ct}}$ method. All experiments were performed in triplicate and the averages were calculated, with GAPDH used as the internal reference. The primers used were as follows: AK058003 forward, 5'-CAGATGGCTGAGGTGGAAGG-3' and reverse, 5'-GACAAGGTCTCGCTCTTTTGCT-3'; SNCG forward, 5'-CACCCTCTGGTCCTTCTG-3' and reverse, 5'-AGGAGTGGGCTCAAGTTT-3'; GAPDH forward, 5'-AGGTGAAGGTCGGAGTCAAC-3' and reverse, 5'-CGCTCCTGGAAGATGGTGAT-3'. The conditions for PCR were as follows: $95^{\circ} \mathrm{C}$ for $1 \mathrm{~min}, 95^{\circ} \mathrm{C}$ for $15 \mathrm{sec}, 60^{\circ} \mathrm{C}$ for $30 \mathrm{sec}, 72^{\circ} \mathrm{C}$ for $15 \mathrm{sec}$, for a total of 40 cycles, followed by $72^{\circ} \mathrm{C}$ for $5 \mathrm{~min}$.

Western blot analysis. Following liposome transfection for $72 \mathrm{~h}$, cells from the control, interference, mock and NC groups were washed twice with cold phosphate-buffered saline, and radioimmunoprecipitation assay lysis buffer was added for total protein extraction. Sodium dodecyl sulfate-polyacrylamide gel electrophoresis was performed to separate the proteins, which were subsequently transferred onto a polyvinylidene difluoride membrane. The membrane was incubated with appropriate concentrations of primary antibodies (SNCG, 1:1,000; GAPDH, 1:2,000; Bioworld Technology Inc., St. Louis Park, MN, USA) overnight at $4^{\circ} \mathrm{C}$. After washing the membrane with phosphate-buffered saline with Tween-20 three times for $15 \mathrm{~min}$, the membrane was incubated with horseradish peroxidase-conjugated secondary antibodies (goat anti-mouse, 1:3,000, \#BS11502; goat anti-rabbit, 1:1,000, \#BS12478; all from Bioworld Technology Inc.) for $1 \mathrm{~h}$ at room temperature. Following three washes with phosphate-buffered saline with Tween-20 for $15 \mathrm{~min}$, the membrane was visualized using an enhanced chemiluminescence detection kit (Sigma-Aldrich, St. Louis, MO, USA) for imaging. Image $\mathrm{Lab}^{\mathrm{TM}}$ software (Bio-Rad, Hercules, CA, USA) was used to acquire and analyze the imaging signals. The relative content of the SNCG protein was expressed as the SNCG/GAPDH ratio.

\section{3-(4,5-dimethylthiazol-2-yl)-2,5-diphenyltetrazolium bromide} (MTT) assay. Cells were seeded into 96-well plates at a density of 3,000 cells/well. Next, $20 \mu 1$ MTT ( $5 \mathrm{mg} / \mathrm{ml}$; Sigma-Aldrich) was added to each well prior to incubation for $4 \mathrm{~h}$ at $37^{\circ} \mathrm{C}$, 
A

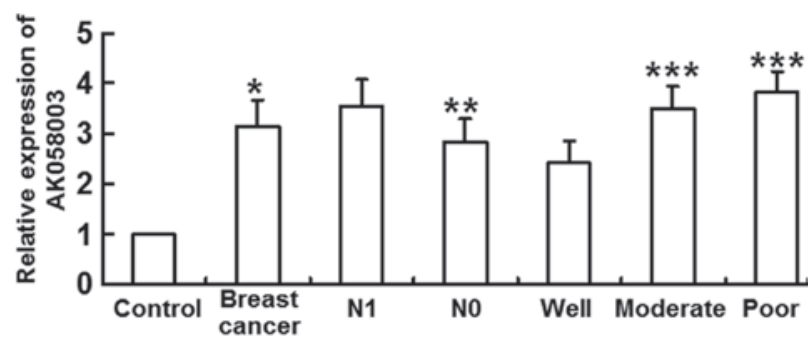

B

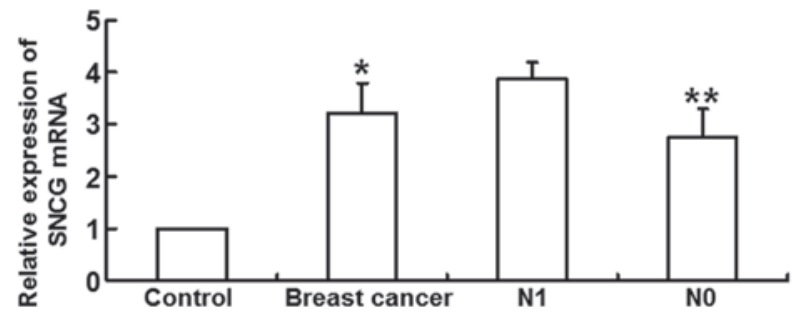

Figure 1. Relative expression levels of (A) long non-coding RNA-AK058003 and (B) SNCG mRNA in breast cancer tissues. Data are expressed as the mean \pm standard deviation. ${ }^{*} \mathrm{P}<0.05$, vs. control group; ${ }^{* *} \mathrm{P}<0.05$, vs. N1 group; ${ }^{* * * *} \mathrm{P}<0.05$, vs. well-differentiated group. SNCG, $\gamma$-synuclein gene; N1, lymph node metastasis group; N0, lymph node non-metastasis group; well, well-differentiated group; moderate, moderately-differentiated group; poor, poorly-differentiated group.

A

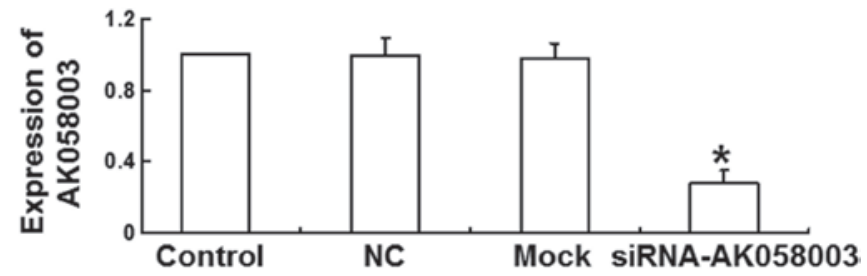

B
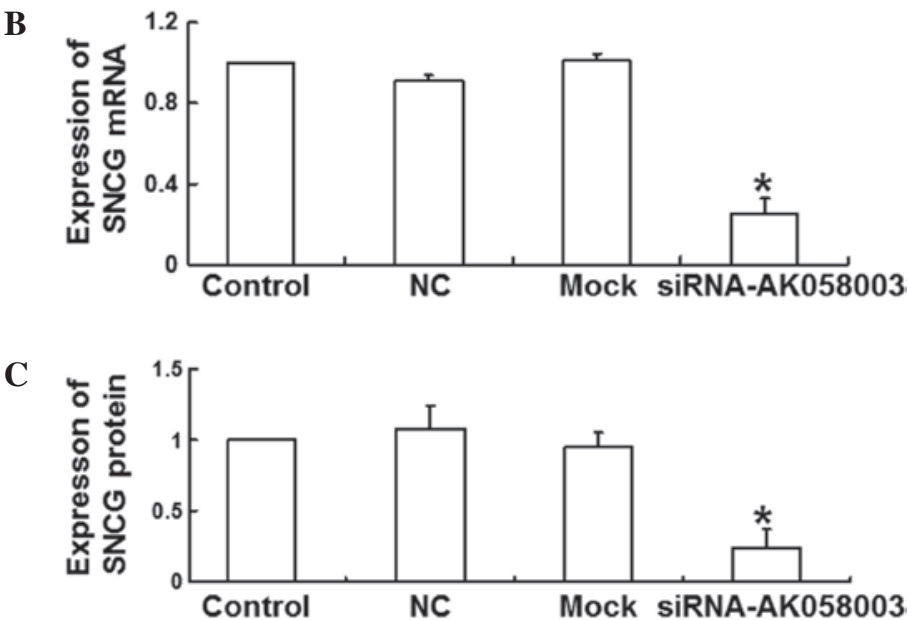

Figure 2. Expression levels of (A) long non-coding RNA-AK058003, (B) SNCG mRNA and (C) SNCG protein in MCF-7 cells at $48 \mathrm{~h}$ after treatment with siRNA-AK058003. Expression levels of mRNA were determined using quantitative polymerase chain reaction, while protein expression levels were measured using western blot analysis. Data are expressed as the mean \pm standard deviation. ${ }^{*} \mathrm{P}<0.05$, vs. NC or mock groups. SNCG, $\gamma$-synuclein gene; NC, negative control group; siRNA, small interfering RNA.

which was followed by the addition of $150 \mu \mathrm{l}$ dimethyl sulfoxide. After $4 \mathrm{~h}$ of incubation, the absorbance of each well was measured at $490 \mathrm{~nm}$ (Multiskan ${ }^{\mathrm{Tu}} \mathrm{GO}$ reader; Thermo Fisher Scientific). A cell proliferation curve was plotted using optical density values.

Transwell ${ }^{\circledR}$ chamber assay. Matrigel (BD Biosciences, Franklin Lakes, NJ, USA) was thawed at $-4^{\circ} \mathrm{C}$ overnight and diluted with serum-free RPMI-1640 medium (dilution, 1:2). A 50- $\mu 1$ sample of this mixture was deposited evenly into a 24 -well chamber $(8 \mu \mathrm{m})$, which was incubated for $60 \mathrm{~min}$ at $37^{\circ} \mathrm{C}$. Upon solidifying, $2 \times 10^{5}$ MCF-7 cells from each group were seeded into the upper chamber containing $300 \mu$ l serum-free RPMI-1640 medium. In addition, $500 \mu 1$ RPMI-1640 medium supplemented with $10 \%$ fetal bovine serum was added to the lower chamber. The upper chamber was removed after $24 \mathrm{~h}$ and the cells in the upper chamber were discarded. The membrane was fixed with $4 \%$ formaldehyde for $10 \mathrm{~min}$ and stained using the Giemsa method for microscopic observation of five random fields (magnification, x200; Olympus IX83; 


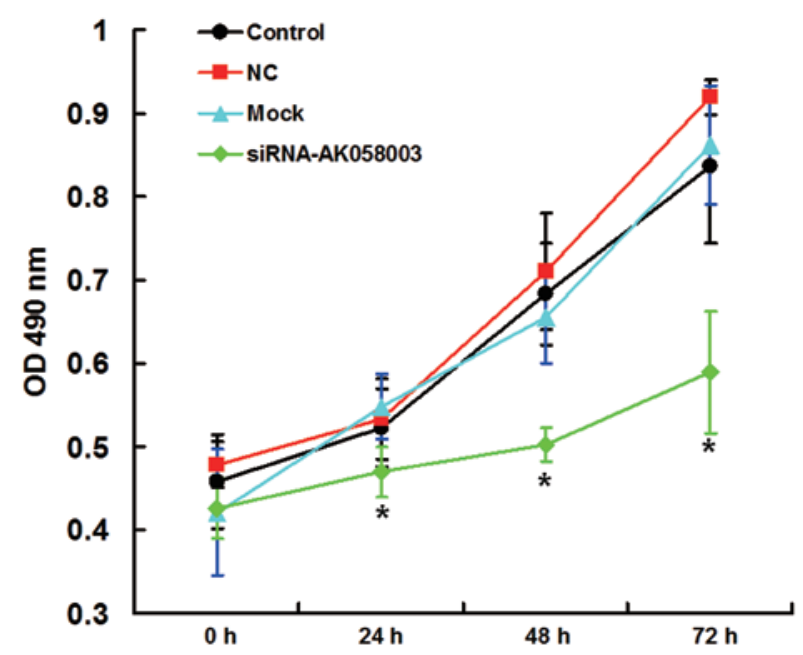

Figure 3. Proliferation curves of MCF-7 cells in the control, mock, NC and siRNA-AK058003 groups, as determined using a 3-(4,5-dimethylthiazol-2-yl)-2,5-diphenyltetrazolium bromide assay and measuring the $\mathrm{OD}$ at $490 \mathrm{~nm}$. Data are expressed as the mean \pm standard deviation. "P<0.05, vs. control, NC or mock groups. NC, negative control group; OD, optical density; siRNA, small interfering RNA.

A

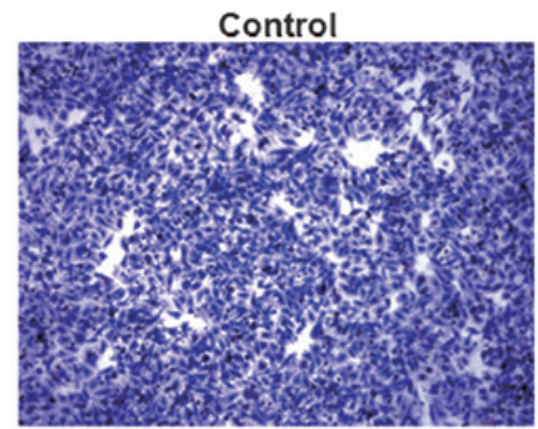

Mock

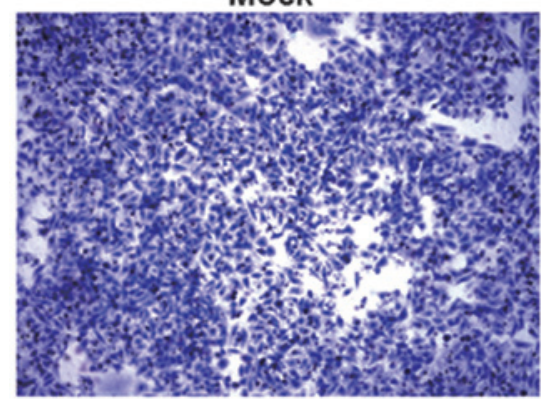

NC

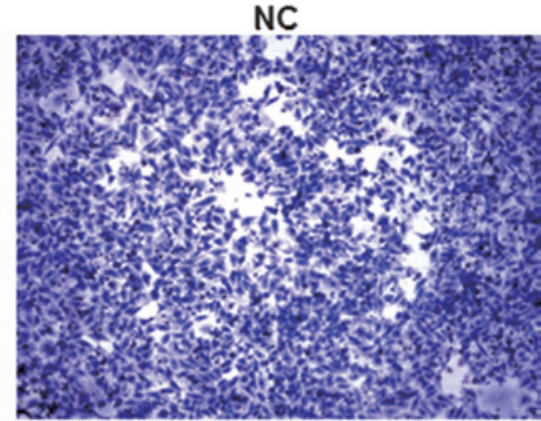

SIRNA-AK058003

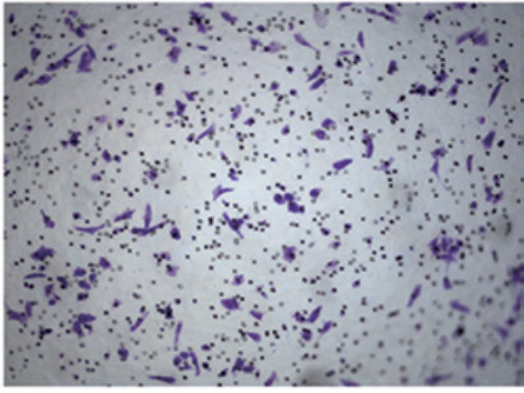

B

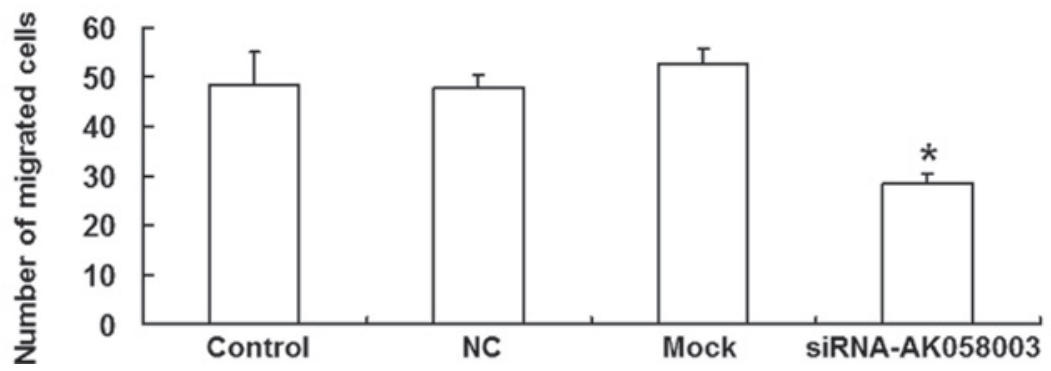

Figure 4. Migration and invasion ability of MCF-7 cells in the control, mock, NC and siRNA-AK058003 groups, as determined by a Transwell ${ }^{\circledR}$ assay. (A) Microscopic observation of MCF-7 cells (Giemsa stain; magnification, x200). (B) Quantification of MCF-7 cells passing through the membrane. Data are expressed as the mean \pm standard deviation. "P<0.05, vs. control, $\mathrm{NC}$ or mock groups. NC, negative control group; siRNA, small interfering RNA.

Olympus Corporation, Tokyo, Japan), from which the number of cells that had migrated was calculated. All procedures were conducted on ice using pipetting tips cooled to $4^{\circ} \mathrm{C}$.
Statistical analysis. Data were analyzed using SPSS 16.0 software (SPSS, Inc., Chicago, IL, USA). Measurement data are expressed as the mean \pm standard deviation. The $\mathrm{t}$-test 
was used for comparisons between groups, where $\mathrm{P}<0.05$ was considered to indicate a statistically significant difference.

\section{Results}

LncRNA-AK058003 and SNCG mRNA expression levels are enhanced in breast cancer tissue. qPCR was employed to measure the expression levels of IncRNA-AK058003 and SNCG mRNA. The results revealed that relative lncRNA-AK058003 expression levels were significantly higher in the breast cancer tissues $(3.14 \pm 1.05$ times) when compared with the adjacent tissues $(\mathrm{P}<0.05)$. In addition, IncRNA-AK058003 expression levels in the N1 group (3.55 \pm 1.04$)$ were significantly higher when compared with the N0 group $(2.82 \pm 0.96 ; \mathrm{P}<0.05)$. LncRNA-AK058003 expression in the well-differentiated group $(2.42 \pm 0.89)$ was significantly lower than that in the moderate $(3.51 \pm 0.86)$ and poor differentiation groups $(3.82 \pm 0.82$; $\mathrm{P}<0.05)$, with the moderate differentiation group exhibiting no statistically significant difference when compared with the poor differentiation group ( $\mathrm{P}>0.05$; Fig. 1A). In addition, the relative SNCG mRNA expression levels increased significantly in the breast cancer tissues (3.22 \pm 0.56 times) when compared with the adjacent tissues $(\mathrm{P}<0.05)$. Relative SNCG mRNA expression levels were higher in the N1 group (3.87 \pm 0.37 times) when compared with the N0 group (P>0.05; Fig. 1B). These observations indicated that lncRNA-AK058003 and SNCG mRNA expression levels were elevated in breast cancer tissues.

Expression of IncRNA-AK058003 is inhibited by AK058003 siRNA. LncRNA-AK058003 expression levels were determined $48 \mathrm{~h}$ after siRNA transfection in MCF-7 cells in order to analyze the effect of AK058003 siRNA on the expression of IncRNA-AK058003. The results revealed that siRNA transfection significantly reduced the expression levels of IncRNA-AK058003 when compared with the NC and mock groups $(\mathrm{P}<0.05$; Fig. 2A). These data demonstrated that the expression of IncRNA-AK058003 was inhibited by AK058003 siRNA.

SNCG $m R N A$ and protein expression levels are reduced by AK058003 siRNA. qPCR and western blot analysis were performed to investigate the effects of AK058003 siRNA on the mRNA and protein expression levels of SNCG. The results of $\mathrm{qPCR}$ revealed that the relative SNCG mRNA expression levels decreased significantly at $48 \mathrm{~h}$ after transfection with AK058003 siRNA (0.25 \pm 0.08 times of control; $\mathrm{P}<0.05$; Fig. $2 \mathrm{~B}$ ). Consistent with this result, relative SNCG protein expression levels also decreased significantly $(0.24 \pm 0.13$ times of control; $\mathrm{P}<0.05$; Fig. $2 \mathrm{C})$, indicating that SNCG mRNA and protein expression levels were reduced by AK058003 siRNA.

MCF-7 cell proliferation is inhibited by AK058003 siRNA. An MTT assay was performed to assess the effects of AK058003 siRNA on MCF-7 cell proliferation. Cell proliferation in the interference group was significantly reduced when compared with the control, NC and mock groups at 24, 48 and $72 \mathrm{~h}$ following the AK058003 siRNA treatment $(\mathrm{P}<0.05$; Fig. 3), indicating that $\mathrm{MCF}-7$ cell proliferation was inhibited by AK058003 siRNA.

MCF-7 cell migration and invasion are reduced by AK058003 siRNA. A Transwell ${ }^{\circledR}$ chamber assay was employed to assess the effects of AK058003 siRNA on MCF-7 cell migration. The number of MCF-7 cells that passed through the membrane was significantly reduced in the AK058003 siRNA group $(28.6 \pm 1.75 ; \mathrm{P}<0.05)$ when compared with the control (48.4 \pm 6.49$), \mathrm{NC}(47.8 \pm 2.65)$ and mock groups (52.4 \pm 3.16 ; Fig. 4). These results indicated that MCF-7 cell migration and invasion were reduced by AK058003 siRNA.

\section{Discussion}

Mammalian genomes encode hundreds of lncRNAs with numerous mechanisms of action. Notably, lncRNAs play an important role in dosage compensation, epigenetics, the cell cycle and differentiation (12). The primary functions of lncRNA include genomic imprinting, chromatin remodeling, mRNA transcriptional regulation and cell cycle regulation (13). The present study determined the expression levels of lncRNA-AK058003 in breast cancer tissue and the potential correlation with the expression of SNCG, which regulates MCF-7 breast cancer cells. LncRNA-AK058003 expression levels were found to be significantly increased in the breast cancer tissue, and particularly in cases that had lymph node metastasis. The well-differentiated group exhibited significantly lower IncRNA-AK058003 expression levels when compared with the moderate and poor differentiation groups. Thus, the level of 1ncRNA-AK058003 expression was demonstrated to be closely associated with the extent of lymph node metastasis and cell differentiation. In addition, SNCG expression levels were significantly increased in the breast cancer tissue and correlated positively with lymph node metastasis. Downregulation of lncRNA-AK058003 using siRNA in breast cancer MCF-7 cells significantly reduced the level of SNCG expression. Furthermore, MTT and Transwell ${ }^{\circledR}$ assays demonstrated that the proliferation, invasion and migration abilities of the MCF-7 cells were significantly reduced by the downregulation of IncRNA-AK058003 expression. These findings demonstrated that IncRNA-AK058003 regulated SNCG expression, possibly by affecting the SNCG methylation levels. However, the exact mechanism underlying this effect requires further study.

In summary, lncRNA-AK058003 expression levels were demonstrated to be significantly increased in breast cancer tissues. Furthermore, lncRNA-AK058003 expression was shown to promote breast cancer proliferation, invasion and metastasis by regulating SNCG expression, which may prove useful for the early diagnosis and prognosis of breast cancer patients.

\section{Acknowledgements}

The authors thank Dr Yongzheng Min from the Department of Urology at the General Hospital of Jinan Military Region (Jinan, China) for valuable suggestions and instructions during this study. 


\section{References}

1. Depla AL, Scharloo-Karels CH, de Jong MA, et al: Treatment and prognostic factors of radiation-associated angiosarcoma (RAAS) after primary breast cancer: a systematic review. Eur J Cancer 50: 1779-1788, 2014.

2. Harbeck N, Beckmann MW, Rody A, et al: HER2 dimerization inhibitor pertuzumab - mode of action and clinical data in breast cancer. Breast Care (Basel) 8: 49-55, 2013.

3. Koul HK, Pal M and Koul S: Role of p38 MAP kinase signal transduction in solid tumors. Genes Cancer 4: 342-359, 2013.

4. Plaza-Menacho I, Mologni L and McDonald NQ: Mechanisms of RET signaling in cancer: current and future implications for targeted therapy. Cell Signal 26: 1743-1752, 2014.

5. Lianos GD, Vlachos K, Zoras O, Katsios C, Cho WC and Roukos DH: Potential of antibody-drug conjugates and novel therapeutics in breast cancer management. Onco Targets Ther 7 : 491-500, 2014.

6. Peschansky VJ and Wahlestedt C: Non-coding RNAs as direct and indirect modulators of epigenetic regulation. Epigenetics 9: 3-12, 2014.

7. Kornfeld JW and Brüning JC: Regulation of metabolism by long, non-coding RNAs. Front Genet 5: 57, 2014.

8. Negishi M, Wongpalee SP, Sarkar S, et al: A new lncRNA, APTR, associates with and represses the CDKN1A/p21 promoter by recruiting polycomb proteins. PLoS One 9: e95216, 2014.
9. Wang P, Xue Y,Han Y, et al: The STAT3-binding long noncoding RNA lnc-DC controls human dendritic cell differentiation. Science 344: 310-313, 2014

10. Ye ZQ, Wang $\mathrm{T}$ and Song $\mathrm{W}$ : Long noncoding RNAs in prostate cancer. Zhonghua Nan Ke Xue 20: 963-968, 2014 (In Chinese)

11. Huang L, Liao LM, Liu AW, Wu JB, Cheng XL, Lin JX and Zheng M: Overexpression of long noncoding RNA HOTAIR predicts a poor prognosis in patients with cervical cancer. Arch Gynecol Obstet 290: 717-723, 2014

12. Rossi MN and Antonangeli F: LncRNAs: New players in apoptosis control. Int J Cell Biol 2014: 473857, 2014.

13. Hrdlickova B, de Almeida RC, Borek Z and Withoff S: Genetic variation in the non-coding genome: Involvement of micro-RNAs and long non-coding RNAs in disease. Biochim Biophys Acta 1842: 1910-1922, 2014.

14. Singh VK and Jia Z: Targeting synuclein- $\gamma$ to counteract drug resistance in cancer. Expert Opin Ther Targets 12: 59-68, 2008.

15. Chen J, Huang S, Wu KJ, et al: The correlation of synuclein- $\gamma$ and matrix metalloproteinase 9 in breast cancer. Zhonghua Wai Ke Za Zhi 51: 641-644, 2013 (In Chinese).

16. Luo JH, Zhou J and Gao Y: Correlation between periostin and SNCG and esophageal cancer invasion, infiltration and apoptosis. Asian Pac J Trop Med 6: 516-519, 2013.

17. Lu A, Zhang F, Gupta A and Liu J: Blockade of AP1 transactivation abrogates the abnormal expression of breast cancer-specific gene 1 in breast cancer cells. J Biol Chem 277: 31364-31372, 2002. 